\title{
Article \\ RNA-Based Assay for Next-Generation Sequencing of Clinically Relevant Gene Fusions in Non-Small Cell Lung Cancer
}

\author{
Caterina De Luca ${ }^{1,+}{ }^{\oplus}$, Francesco Pepe ${ }^{1,+}$, Antonino Iaccarino ${ }^{1,+}$, Pasquale Pisapia ${ }^{1} \mathbb{C}$, Luisella Righi ${ }^{2}$, \\ Angela Listì ${ }^{2}$, Lorenza Greco ${ }^{1}$, Gianluca Gragnano ${ }^{1}$, Severo Campione ${ }^{3}$, Gianfranco De Dominicis ${ }^{3}$, \\ Fabio Pagni ${ }^{4}{ }^{\mathbb{D}}$, Roberta Sgariglia ${ }^{1}$, Mariantonia Nacchio ${ }^{1}$, Rossella Tufano ${ }^{5}$, Floriana Conticelli ${ }^{1}$,

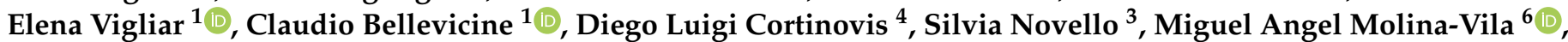 \\ Rafael Rosell ${ }^{7}$, Giancarlo Troncone ${ }^{1, *} *$ and Umberto Malapelle ${ }^{1}$ (D)
}

check for updates

Citation: De Luca, C.; Pepe, F.; Iaccarino, A.; Pisapia, P.; Righi, L.; Listì, A.; Greco, L.; Gragnano, G.; Campione, S.; De Dominicis, G.; et al. RNA-Based Assay for Next-Generation Sequencing of Clinically Relevant Gene Fusions in Non-Small Cell Lung Cancer. Cancers 2021, 13, 139. https:// doi.org/10.3390/cancers13010139

Received: 10 December 2020

Accepted: 2 January 2021

Published: 4 January 2021

Publisher's Note: MDPI stays neutral with regard to jurisdictional clai$\mathrm{ms}$ in published maps and institutional affiliations.

Copyright: () 2021 by the authors. Licensee MDPI, Basel, Switzerland. This article is an open access article distributed under the terms and conditions of the Creative Commons Attribution (CC BY) license (https:// creativecommons.org/licenses/by/ $4.0 /)$.
1 Department of Public Health, University of Naples Federico II, 80131 Naples, Italy; caterina.deluca@unina.it (C.D.L.); francesco.pepe4@unina.it (F.P.); antiaccc@hotmail.com (A.I.); pasquale.pisapia@unina.it (P.P.); lorenza.greco6@gmail.com (L.G.); gianluca.gragnano@unina.it (G.G.); roberta.sgariglia@unina.it (R.S.); mariantonia.nacchio@unina.it (M.N.); floriana.conticelli@unina.it (F.C.); elena.vigliar@unina.it (E.V.); claudio.bellevicine@unina.it (C.B.); umberto.malapelle@unina.it (U.M.)

2 Department of Oncology, San Luigi University Hospital, University of Turin, 10043 Orbassano, Italy; luisella.righi@unito.it (L.R.); alisti@live.it (A.L.)

3 Anatomic Pathology, A.O.R.N. Antonio Cardarelli, 80131 Naples, Italy; severo.campione@aocardarelli.it (S.C.); gianfranco.dedominicis@aocardarelli.it (G.D.D.); silvia.novello@unito.it (S.N.)

4 Department of Medicine and Surgery, San Gerardo Hospital, University of Milano-Bicocca, 20900 Monza, Italy; fabio.pagni@unimib.it (F.P.); d.cortinovis@asst.monza.it (D.L.C.)

5 CEINGE Biotecnologie Avanzate, Via Gaetano Salvatore 486, 80131 Naples, Italy; rossella.tufano@libero.it

6 Laboratory of Oncology, Pangaea Oncology, 08028 Barcelona, Spain; mamolina@panoncology.com

7 Cancer Biology and Precision Medicine Program Catalan Institute of Oncology,

Germans Trias i Pujol Health Sciences Institute and Hospital Badalona, 08916 Barcelona, Spain; rrosell@iconcologia.net

* Correspondence: giancarlo.troncone@unina.it; Fax: +39-(011)-0817-463-679

+ These Authors contributed equally.

Simple Summary: Gene fusions represent novel predictive biomarkers for advanced Non Small Cell Lung Cancer (NSCLC) patients. In this study, we developed and validated a narrow Next Generation Sequencing gene panel able to cover ALK, ROS1, RET and NTRK gene fusions and MET splicing events in advanced-stage NSCLC patients. Overall, our RNA fusion panel was able to detect all fusions and a splicing event harbored in a RNA pool diluted up to $2 \mathrm{ng} / \mu \mathrm{L}$. In addition, It also successfully analyzed 46 (95.8\%) out of 48 routine samples previously characterized by conventional non - NGS technology, representing a robust tool for routine setting.

Abstract: Gene fusions represent novel predictive biomarkers for advanced non-small cell lung cancer (NSCLC). In this study, we validated a narrow NGS gene panel able to cover therapeuticallyrelevant gene fusions and splicing events in advanced-stage NSCLC patients. To this aim, we first assessed minimal complementary DNA (cDNA) input and the limit of detection (LoD) in different cell lines. Then, to evaluate the feasibility of applying our panel to routine clinical samples, we retrospectively selected archived lung adenocarcinoma histological and cytological (cell blocks) samples. Overall, our SiRe RNA fusion panel was able to detect all fusions and a splicing event harbored in a RNA pool diluted up to $2 \mathrm{ng} / \mu \mathrm{L}$. It also successfully analyzed $46(95.8 \%)$ out of 48 samples. Among these, 43 (93.5\%) out of 46 samples reproduced the same results as those obtained with conventional techniques. Intriguingly, the three discordant results were confirmed by a CE-IVD automated real-time polymerase chain reaction (RT-PCR) analysis (Easy PGX platform, Diatech Pharmacogenetics, Jesi, Italy). Based on these findings, we conclude that our new SiRe RNA fusion panel is a valid and robust tool for the detection of clinically relevant gene fusions and splicing events in advanced NSCLC. 
Keywords: predictive molecular pathology; next generation sequencing; gene fusions; targeted therapy; NSCLC

\section{Introduction}

Non-small cell lung cancer (NSCLC) represents the leading cause of cancer mortality worldwide [1]. Unfortunately, being commonly diagnosed in the advanced stages of the disease, it is very difficult to treat. Over the last decade, an upturn in NSCLC treatment has been the identification and use of several biomarkers to predict patients' response to personalized treatments. Indeed, biomarker-based personalized treatments have significantly improved progression free survival (PFS) in advanced stage NSCLC patients harboring several actionable point mutations, indels, and gene rearrangements [2-8]. One such biomarker is Anaplastic Lymphoma Kinase $(A L K)$ rearrangement (3-6\%), which can predict marked sensitivity to crizotinib, alectinib, and brigatinib treatments [5,9-14]. Another important biomarker is ROS Proto-Oncogene 1 Receptor Tyrosine Kinase (ROS1) gene fusion (1-2\%), which is known to respond remarkably well to crizotinib [15-17]. Similarly, Neurotrophic Receptor Tyrosine Kinase (NTRK) fusions $(<1 \%)$ respond to entrectinib [18-21]. Lastly, REarranged during Transfection (RET) gene fusions (1-2\%) [22,23] respond well to selpercatinib and pralsetinib [24,25].

However, detecting therapeutically relevant gene fusions may be time-consuming and costly, and, in some specific cases, may require high-quality histological samples. For instance, fluorescence in situ hybridization (FISH) and immunohistochemistry (IHC) require a series of consecutive assays to test for all these gene fusions, and, in the case of RET fusion samples, IHC does not yield accurate results.

Conversely, next generation sequencing (NGS) comprehensively and simultaneously evaluates all actionable targets in a single run. [26] Furthermore, besides specifying fusion variants, it can also detect de novo gene fusions and other RNA alterations, including mutations in the MET Proto-Oncogene, Receptor Tyrosine Kinase (MET) exon 14 skipping isoform [27-29]. However, one drawback of the "wide" NGS gene panels for NSCLC is that they comprise not only vast numbers of actionable standard of care targets but also extrapulmonary and investigational DNA and/or RNA biomarkers, thus rendering these panels rather impracticable in routine clinical practice. Conversely, "narrow" gene panels, [30] which cover only NSCLC targets make NGS more cost-effective and much easier to implement in routine practice. Not surprisingly, there is a much higher request for narrow targeted panels than for larger ones [3].

In our routine clinical practice, we have recently adopted a "narrow" DNA custom gene panel to sequence several somatic mutations, in particular Epidermal Growth Factor Receptor (EGFR), Kirsten Rat Sarcoma Viral Oncogene Homolog (KRAS), Neuroblastoma RAS Viral Oncogene Homolog (NRAS), and V-Raf Murine Sarcoma Viral Oncogene Homolog $\mathrm{B}(B R A F)$ [31]. Moreover, considering that gene fusions and splicing events are equally important cancer driver mutations in NSCLC, we sought to improve our diagnostic performance by adopting the same approach to design a "narrow" RNA custom gene panel, which we called SiRe fusion, specially designed to detect the most clinically relevant gene fusion and splicing events in advanced-stage lung cancer. Thus, the aim of the present study was to validate our novel "narrow" RNA custom gene panel for NGS analysis of $A L K$, ROS1, NTRK, and RET rearrangements, as well as the MET exon 14 skipping mutation.

\section{Materials and Methods}

\subsection{Panel Design and Library Preparation Protocol}

To cover ALK, ROS1, RET, and NTRK gene fusions, as well as and MET exon 14 skipping alterations simultaneously, we developed a fusion primer pool by using AmpliSeq designer software v.7.4 which uses hg19 as a reference sequence. The panel, named SiRe fusion, was designed to sequence cDNA obtained after retro-transcription of RNA extracted 
from formalin fixed and paraffin embedded (FFPE) sections, cytopathological specimens, and body fluid samples of NSCLC patients. Several experimental and analytical steps were carried out to assess the reference range, the minimal input of cDNA, the limit of detection $(\mathrm{LoD})$, and the overall practicability of this panel in routine practice.

Libraries were constructed and purified on the Ion Chef Instrument (ThermoFisher Scientifics, Waltham, MA, USA) according to the manufacturer's instructions. In brief, $6 \mu \mathrm{L}$ of cDNA (with an optimal concentration of $2 \mathrm{ng} /$ microliter) was dispensed on the Ion Code plates and amplified with Ion AmpliSeq DL8 (ThermoFisher Scientifics). cDNA was amplified with 24 cycles, whereas the library was re-amplified with 7 PCR cycles after barcoding, under the thermal conditions recommended by the manufacturer. Purified libraries from RNA samples were diluted to $60 \mathrm{pM}$ and pooled. They were then re-loaded onto the Ion Chef Instrument, and the templates were prepared with the S5 510-520-530 Kit-Chef (ThermoFisher Scientifics). Finally, templates were loaded onto the 520 chip and sequenced on the S5 NGS platform (ThermoFisher Scientifics). The data were interpreted with a proprietary pipeline, developed by the Department of Public Health, University of Naples Federico II, and visualized on Ion Reporter Software (ThermoFisher Scientifics). The bioinformatic analysis was carried out by using Ion Reporter Ion Reporter ${ }^{\mathrm{TM}}$ 5.16.0.2 with customized version of AmpliSeq RNA Lung Fusion-w1.2-Single Sample. All the panel design files were reported in the Supplementary Material (Supplementary Files S1-S9). Any additional information on the panel design and data analysis is available on request.

\subsection{Molecular Reference Standards}

The custom RNA fusion gene panel was validated as follows. Minimal cDNA input and LoD were assesses by employing, different molecular reference standards. Ethanol fixed slides prepared from cells simultaneously harboring Echinoderm Microtubule-Associated Protein-Llike 4 [EML4](10)-ALK(20), Solute Carrier Family 34 Member 2 [SLC34A2](4)ROS1(32), Coiled-Coil Domain Containing 6 [CCDC6](1)-RET(12) and Tropomyosin 3 [TPM3](8)-NTRK1(10)] were purchased from Horizon Diagnostics (HDx Cambridge, United Kingdom). In addition, several cell lines, each carrying a specific gene fusion, were purchased from the American Type Culture Collection. RNA was extracted from of the following cell lines: H3122 [EML4(13)-ALK(20)], H2228 [EML4(6)-ALK(20)], Hs746T (MET exon 14 skipping), H596 (MET exon 14 skipping), HCC-78, [SLC34A2(4)-ROS1(32)]; LC2ad [CCDC6(1)-RET(12)], EBC-1, (MET Amplification), SUDHL-1, (Nucleophosmin 1 [NPM1]$A L K$, and NTRK1cl [TPM3(8)-NTRK1(10)] (Supplementary Table S1). Finally, the extracted RNA was used to prepare reference standards at different dilutions (Supplementary Table S2).

\subsection{RNA Extraction, Retrotrascription and Quantification}

For molecular reference standards, RNA was extracted with the All Prep DNA- RNA Mini Kit (Qiagen, Hilden, Germany), according to the manufacturer's instructions, and resuspended in $50 \mu \mathrm{L}$ of RNAsi/DNAsi free water (Ambion, ThermoFisher Scientifics). RNA was directly extracted from the above mentioned cell lines (Pangaea Biotech, Barcelona, Spain) with the High Pure RNA Isolation kit (Roche Diagnostics, Basel, Switzerland) according to the manufacturer's instructions. TapeStation 4200 (Agilent Technologies, Santa Clara, CA, USA), a microfluidic based technology, evaluated the RNA concentration (ng/ $\mu \mathrm{L})$ and RNA integrity number (RIN). The RNA was eluted in $30 \mu \mathrm{L}$ of RNAsi/DNAsi free water (Ambion, ThermoFisher Scientifics). Retro-transcription was carried out with SuperScript IV VILO Master Mix (ThermoFisher Scientifics) according to the manufacturer's instructions. The cDNA was then quantified by using the D1000 Genomic assay on TapeStation 4200 (Agilent Technologies) (Supplementary Figures S1 and S2). Our custom panel NGS analysis was performed as above described; the results were further confirmed by an automated real time polymerase chain reaction (RT-PCR) approach (Easy PGX platform, Diatech Pharmacogenetics, Jesi, Italy) for ALK, ROS1, RET, and NTRK gene fusions, as well as the MET exon 14 skipping mutation. 


\subsection{Validation of the SiRe Fusion Panel in Routine Samples}

To evaluate the performance of our SiRe fusion panel in a clinical setting, we retrospectively selected archived lung adenocarcinoma histological and cytological (cell blocks) samples harboring ALK, ROS1, RET, and NTRK gene fusions, as well as the MET exon 14 skipping mutation. This retrospective analysis was based on previous diagnoses of gene rearrangements involving $A L K, R O S 1$, and RET made by various Italian institutions by immunohistochemistry (IHC), fluorescent in situ hybridization (FISH), and NGS analyses (University of Naples Federico II, Naples, Italy; A.O.R.N. Antonio Cardarelli, Naples, Italy; San Luigi University Hospital, University of Turin, Orbassano, Italy; San Gerardo Hospital, University of Milano-Bicocca, Monza, Italy) S. Sample characteristics and gene alterations are reported in both Table 1 and Figures 1 and 2.

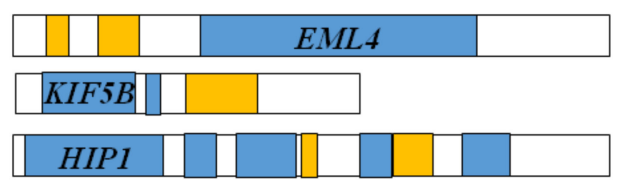

\begin{tabular}{|l|l|l|}
\hline & $A L K$ & \\
\hline & $A L K$ & \\
\hline & $A L K$ & \\
\hline
\end{tabular}

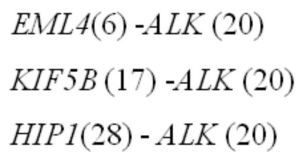

SD PR KD

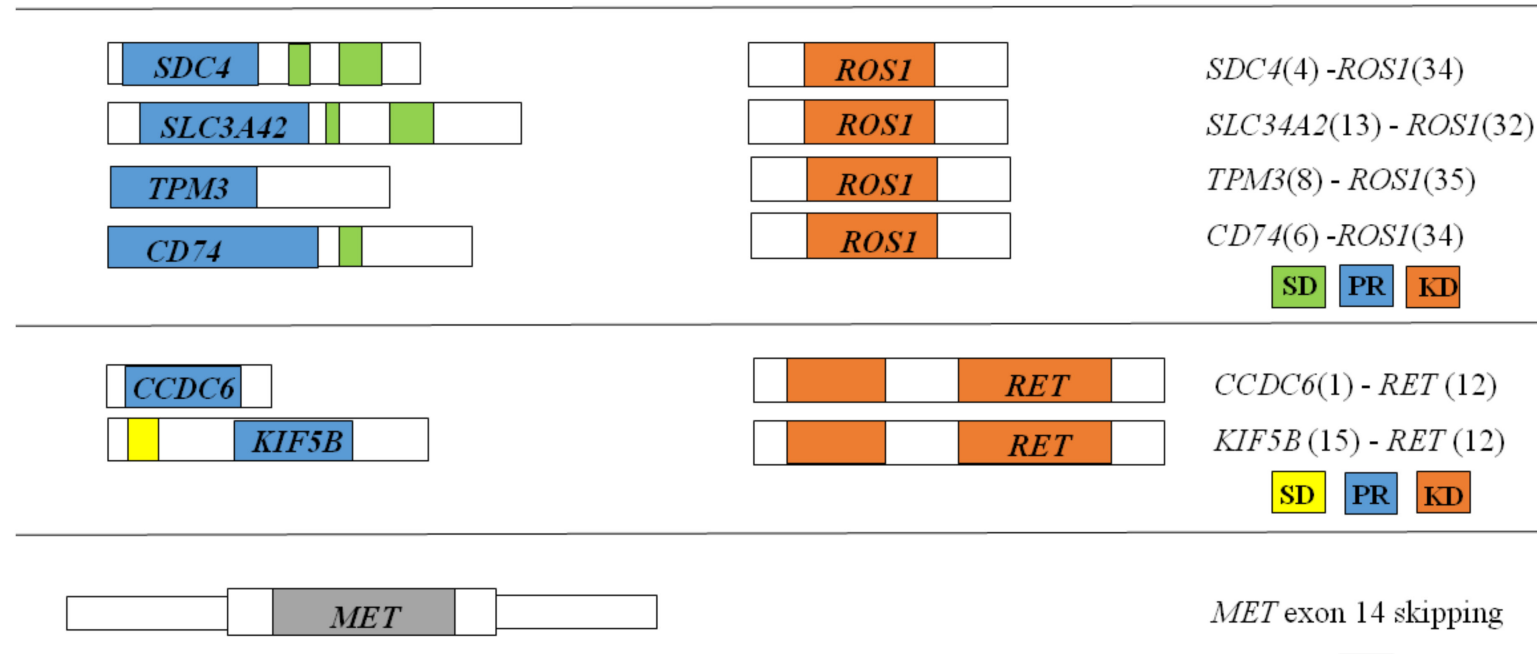

CS

Figure 1. Schematic representation of gene fusions reported in a routine sample set: The kinase domain is in orange; the promoter region is in blue; the structural domains are in yellow, ochre, and green; the coding sequence is in grey. Abbreviations: ALK: Anaplastic Lymphoma Kinase; CCDC6: Coiled-Coil Domain Containing 6; CD74: CD74 Molecule, Major Histocompatibility Complex, Class II Invariant Chain; CS: sequence; EML4: Echinoderm Microtubule Associated Protein Like 4; HIP1: Huntingtin Interacting Protein 1; KD: kinase domain; KIF5B: Kinesin Family Member 5B; MET: MET Proto-Oncogene, Receptor Tyrosine Kinase; PR: promoter region; RET: Rearranged During Transfection; ROS1: ROS Proto-Oncogene 1, Receptor Tyrosine Kinase; SD: structural domain; SLC34A2: Solute Carrier Family 34 Member 2; SDC4: Syndecan 4; TPM3: Tropomyosin 3. 
Table 1. Patients' characteristics and SiRe results.

\begin{tabular}{|c|c|c|c|c|c|c|c|c|c|c|}
\hline \multirow[b]{2}{*}{$\mathbf{N}$} & \multicolumn{5}{|c|}{ Patients and Sample Characteristics } & \multicolumn{2}{|c|}{$\begin{array}{l}\text { Fusions Detected with } \\
\text { Conventional Methods }\end{array}$} & \multicolumn{3}{|c|}{ Fusions Detected with SiRe } \\
\hline & Sex & Age & Sample Type & Sample Sub-Type & $\%$ Neoplastic Cells & Fused Gene & Detection Method & Locus & Genes (Exon) & Reads Count \\
\hline 1 & $\mathrm{~F}$ & 60 & Histological & Resection & $80 \%$ & $A L K$ & $\mathrm{IHC}$ & $\begin{array}{l}\text { chr2:42491871- } \\
\text { chr2:29446394 }\end{array}$ & $E M L 4(6)-A L K(20)$ & 5321 \\
\hline 2 & $\mathrm{M}$ & 62 & Histological & Resection & $60 \%$ & $A L K$ & $\mathrm{IHC}$ & $\begin{array}{l}\text { chr2:42492091- } \\
\text { chr2:29446394 }\end{array}$ & $E M L 4(6)-A L K(20)$ & 6285 \\
\hline 3 & M & 47 & Histological & Resection & $70 \%$ & $A L K$ & $\mathrm{IHC}$ & $\begin{array}{l}\text { chr2:42491871- } \\
\text { chr2:29446394 }\end{array}$ & $E M L 4(6)-A L K(20)$ & 1662 \\
\hline 4 & M & 76 & Histological & Biopsy & $30 \%$ & $A L K$ & $\mathrm{IHC}$ & $\begin{array}{l}\text { chr2:42522656- } \\
\text { chr2:29446394 }\end{array}$ & $E M L 4(13)-A L K(20)$ & 2391 \\
\hline 5 & $\mathrm{~F}$ & 44 & Histological & Resection & $60 \%$ & $A L K$ & $\mathrm{IHC}$ & $\begin{array}{l}\text { chr20:43959006- } \\
\text { chr6:117645578 }\end{array}$ & SDC4(4)-ROS1(34) & 94 \\
\hline 6 & $\mathrm{~F}$ & 63 & Histological & Resection & $70 \%$ & ROS1 & $\mathrm{IHC}$ & $\begin{array}{l}\text { chr5:149784243- } \\
\text { chr6:117645578 }\end{array}$ & CD74(6)-ROS1(34) & 28,678 \\
\hline 7 & $\mathrm{M}$ & 59 & Histological & Biopsy & $60 \%$ & $A L K$ & $\mathrm{IHC}$ & $\begin{array}{l}\text { chr2:42552694- } \\
\text { chr2:29446394 }\end{array}$ & $E M L 4(20)-A L K(20)$ & 4353 \\
\hline 8 & M & 69 & Histological & Biopsy & $40 \%$ & $A L K$ & $\mathrm{IHC}$ & $\begin{array}{l}\text { chr10:32311068- } \\
\text { chr2:29446394 }\end{array}$ & $K I F 5 B(17)-A L K(20)$ & 9641 \\
\hline 9 & $\mathrm{~F}$ & 48 & Histological & Resection & $80 \%$ & ROS1 & $\mathrm{IHC}$ & $\begin{array}{l}\text { chr5:149784243- } \\
\text { chr6:117645578 }\end{array}$ & CD74(6)-ROS1(34) & 4347 \\
\hline 10 & M & 45 & Histological & Biopsy & $40 \%$ & $A L K$ & $\mathrm{IHC}$ & $\begin{array}{l}\text { chr2:42522656- } \\
\text { chr2:29446394 }\end{array}$ & $E M L 4(13)-A L K(20)$ & 5821 \\
\hline 11 & M & 36 & Histological & Resection & $80 \%$ & $A L K$ & $\mathrm{IHC}$ & $\begin{array}{l}\text { chr2:42522656- } \\
\text { chr2:29446394 }\end{array}$ & $E M L 4(13)-A L K(20)$ & 2279 \\
\hline 12 & M & 40 & Histological & Biopsy & $40 \%$ & $A L K$ & IHC & $\begin{array}{l}\text { chr2:42522656- } \\
\text { chr2:29446394 }\end{array}$ & $E M L 4(13)-A L K(20)$ & 1659 \\
\hline 13 & $\mathrm{~F}$ & 40 & Histological & Resection & $80 \%$ & $A L K$ & $\mathrm{IHC}$ & $\begin{array}{l}\text { chr2:42492091- } \\
\text { chr2:29446394 }\end{array}$ & $E M L 4(6)-A L K(20)$ & 349 \\
\hline
\end{tabular}


Table 1. Cont.

\begin{tabular}{|c|c|c|c|c|c|c|c|c|c|c|}
\hline \multirow[b]{2}{*}{$\mathbf{N}$} & \multicolumn{5}{|c|}{ Patients and Sample Characteristics } & \multicolumn{2}{|c|}{$\begin{array}{l}\text { Fusions Detected with } \\
\text { Conventional Methods }\end{array}$} & \multicolumn{3}{|c|}{ Fusions Detected with SiRe } \\
\hline & Sex & Age & Sample Type & Sample Sub-Type & $\%$ Neoplastic Cells & Fused Gene & Detection Method & Locus & Genes (Exon) & Reads Count \\
\hline 14 & $\mathrm{M}$ & 57 & Histological & Biopsy & $70 \%$ & $A L K$ & $\mathrm{IHC}$ & $\begin{array}{l}\text { chr2:42491871- } \\
\text { chr2:29446394 }\end{array}$ & $E M L 4(6)-A L K(20)$ & 4401 \\
\hline 15 & $\mathrm{M}$ & 55 & Histological & Biopsy & $60 \%$ & $A L K$ & IHC & $\begin{array}{l}\text { chr2:42543190- } \\
\text { chr2:29446463 }\end{array}$ & $E M L 4(18)-A L K(20)$ & 460 \\
\hline 16 & $\mathrm{~F}$ & 34 & Cytological & Cell-block & $60 \%$ & $A L K$ & IHC & $\begin{array}{l}\text { chr2:42552694- } \\
\text { chr2:29446394 }\end{array}$ & $E M L 4(20)-A L K(20)$ & 12,782 \\
\hline 17 & $\mathrm{~F}$ & 76 & Cytological & Cell-block & $20 \%$ & $A L K$ & NGS & $\begin{array}{l}\text { chr2:42522656- } \\
\text { chr2:29446394 }\end{array}$ & $E M L 4(13)-A L K(20)$ & 4145 \\
\hline 18 & $\mathrm{~F}$ & 65 & Cytological & Cell-block & $40 \%$ & $A L K$ & FISH & - & Not detected & - \\
\hline 19 & $\mathrm{M}$ & 71 & Cytological & Cell-block & $20 \%$ & $A L K$ & FISH & unknown & ALK (unknown partner) & - \\
\hline 20 & M & 51 & Histological & Resection & $70 \%$ & $A L K$ & FISH plus IHC & $\begin{array}{l}\text { chr2:42492091- } \\
\text { chr2:29446394 }\end{array}$ & $E M L 4(6)-A L K(20)$ & 16,459 \\
\hline 21 & M & 81 & Cytological & Cell-block & $70 \%$ & $A L K$ & FISH plus IHC & $\begin{array}{l}\text { chr2:42491871- } \\
\text { chr2:29446394 }\end{array}$ & $E M L 4(6)-A L K(20)$ & 6005 \\
\hline 22 & $\mathrm{~F}$ & 60 & Cytological & Cell-block & $70 \%$ & $A L K$ & FISH & $\begin{array}{l}\text { chr2:42522656- } \\
\text { chr2:29446394 }\end{array}$ & $E M L 4(13)-A L K(20)$ & 6079 \\
\hline 23 & $\mathrm{M}$ & 46 & Cytological & Cell-block & $40 \%$ & RET & NGS & $\begin{array}{l}\text { chr10:61665880- } \\
\text { chr10:43612032 }\end{array}$ & $C C D C 6(1)-R E T(12)$ & 27,834 \\
\hline 24 & $\mathrm{~F}$ & 67 & Cytological & Cell-block & $20 \%$ & $A L K$ & FISH & - & Not detected & - \\
\hline 25 & $\mathrm{~F}$ & 51 & Cytological & Cell-block & $20 \%$ & $A L K$ & FISH & unknown & $A L K$ (unknown partner) & - \\
\hline 26 & $\mathrm{~F}$ & 49 & Histological & Resection & $70 \%$ & $A L K$ & FISH plus IHC & $\begin{array}{l}\text { chr2:42491871- } \\
\text { chr2:29446394 }\end{array}$ & $E M L 4(6)-A L K(20)$ & 317 \\
\hline
\end{tabular}


Table 1. Cont.

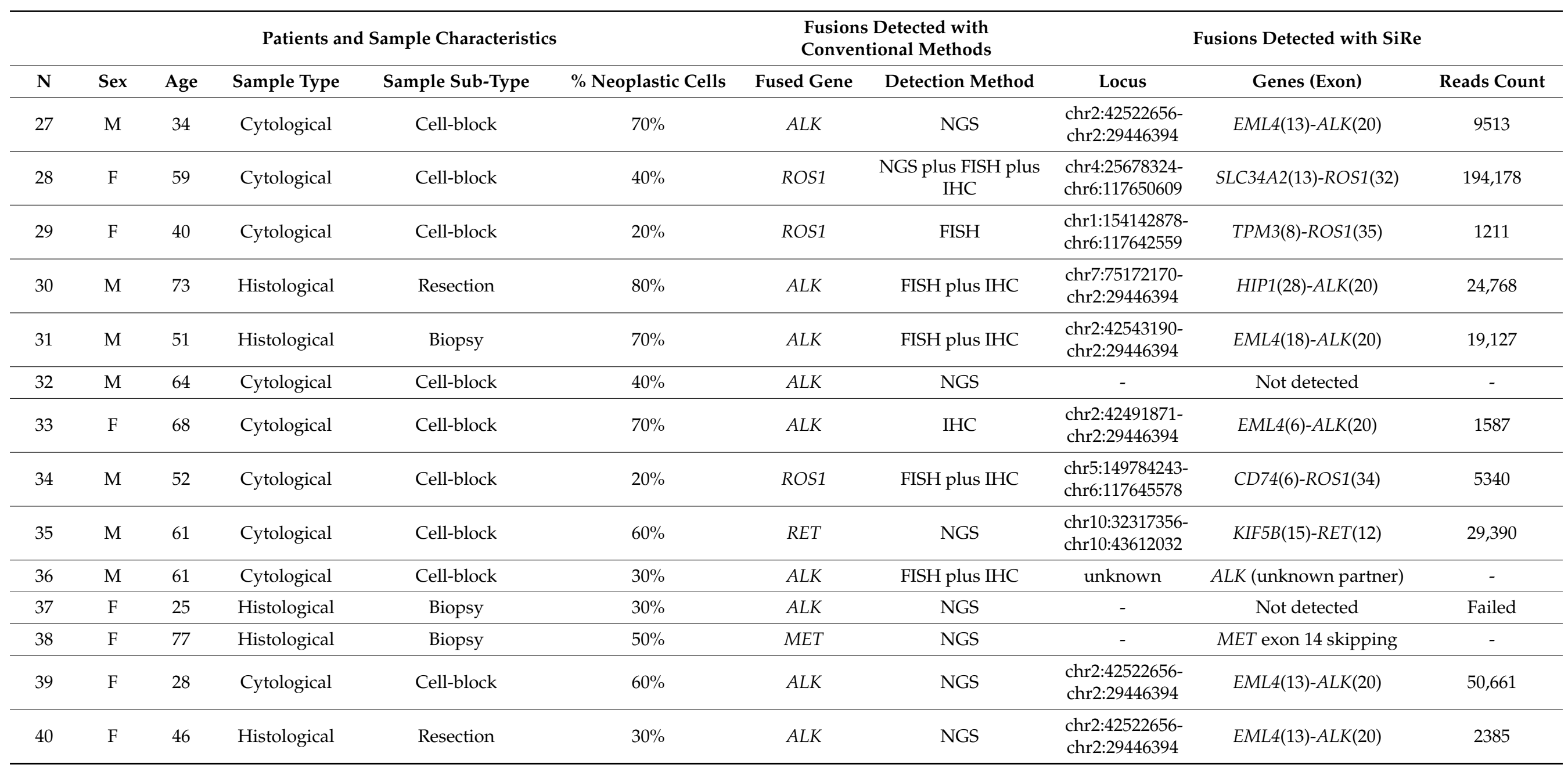


Table 1. Cont.

\begin{tabular}{|c|c|c|c|c|c|c|c|c|c|c|}
\hline \multirow[b]{2}{*}{$\mathbf{N}$} & \multicolumn{5}{|c|}{ Patients and Sample Characteristics } & \multicolumn{2}{|c|}{$\begin{array}{l}\text { Fusions Detected with } \\
\text { Conventional Methods }\end{array}$} & \multicolumn{3}{|c|}{ Fusions Detected with SiRe } \\
\hline & Sex & Age & Sample Type & Sample Sub-Type & $\%$ Neoplastic Cells & Fused Gene & Detection Method & Locus & Genes (Exon) & Reads Count \\
\hline 41 & $\mathrm{~F}$ & 62 & Histological & Resection & $60 \%$ & MET & NGS & - & MET exon 14 skipping & - \\
\hline 42 & M & 57 & Histological & Resection & $60 \%$ & $A L K$ & NGS & $\begin{array}{l}\text { chr2:42522656- } \\
\text { chr2:29446394 }\end{array}$ & $E M L 4(13)-A L K(20)$ & 10,836 \\
\hline 43 & $\mathrm{~F}$ & 74 & Cytological & Cell-block & $40 \%$ & RET & NGS & $\begin{array}{l}\text { chr10:61665880- } \\
\text { chr10:43612032 }\end{array}$ & CCDC6(1)-RET(12) & 15,342 \\
\hline 44 & M & 59 & Histological & Biopsy & $20 \%$ & RET & NGS & $\begin{array}{l}\text { chr10:61665880- } \\
\text { chr10:43612032 }\end{array}$ & $C C D C 6(1)-R E T(12)$ & 24,018 \\
\hline 45 & $\mathrm{~F}$ & 62 & Histological & Biopsy & $20 \%$ & $A L K$ & FISH plus IHC & $\begin{array}{l}\text { chr2:42552694- } \\
\text { chr2:29446394 }\end{array}$ & $E M L 4(20)-A L K(20)$ & 11,823 \\
\hline 46 & M & 62 & Histological & Biopsy & $50 \%$ & $A L K$ & NGS plus IHC & $\begin{array}{l}\text { chr2:42472827- } \\
\text { chr2:29446394 }\end{array}$ & $E M L 4(2)-A L K(20)$ & 53,460 \\
\hline 47 & $\mathrm{~F}$ & 71 & Histological & Resection & $50 \%$ & MET & NGS & - & MET exon 14 skipping & - \\
\hline 48 & M & 48 & Histological & Biopsy & $80 \%$ & $A L K$ & FISH plus IHC & - & Not detected & Failed \\
\hline
\end{tabular}

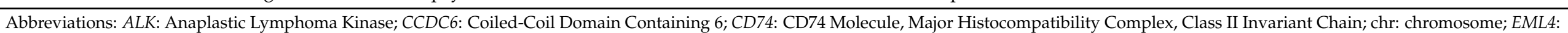

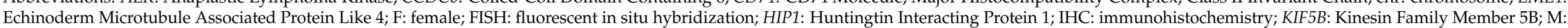

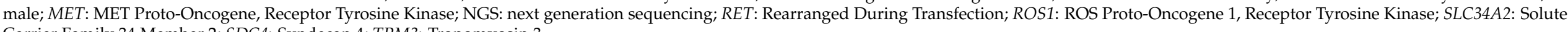
Carrier Family 34 Member 2; SDC4: Syndecan 4; TPM3: Tropomyosin 3. 
A

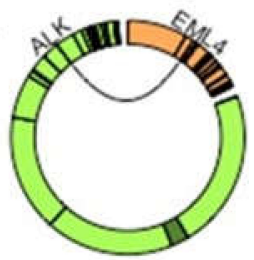

D

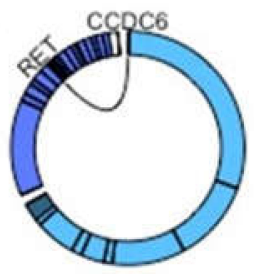

G

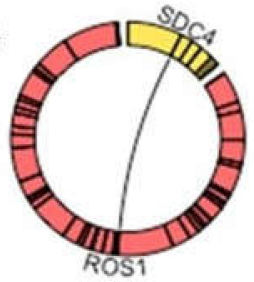

B

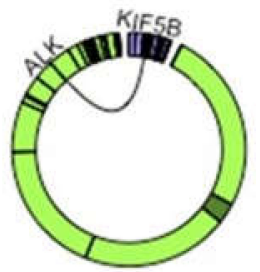

E

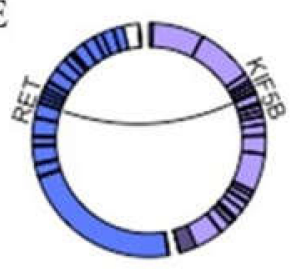

$\mathrm{H}$

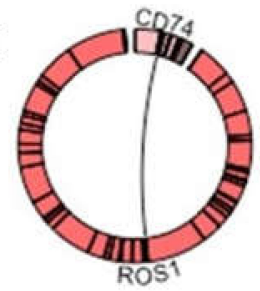

$\mathrm{C}$

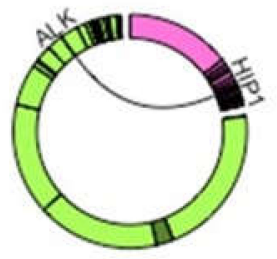

F

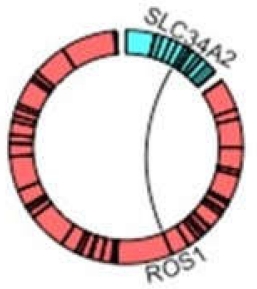

I

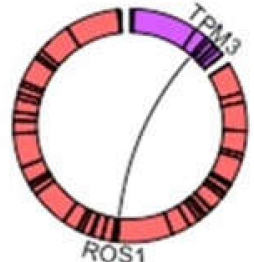

Figure 2. Circos plots representation of the gene fusions reported in routine sample set by using the $\mathrm{R}$ circlize package (R Core Team v3.6.1) [32]. (A-C) shows $A L K$ translocations and corresponding fusion partners detected in analyzed samples. (D,E) shows RET fusions and gene partners identified in sample population. (F-I) shows ROS1 translocations and fusion partners inspected in analyzed samples. Abbreviations: ALK: Anaplastic Lymphoma Kinase; CCDC6: Coiled-Coil Domain Containing 6; CD74: CD74 Molecule, Major Histocompatibility Complex, Class II Invariant Chain; CS: sequence; EML4: Echinoderm Microtubule Associated Protein Like 4; HIP1: Huntingtin Interacting Protein 1; KD: kinase domain; KIF5B: Kinesin Family Member 5B; MET: MET Proto-Oncogene, Receptor Tyrosine Kinase; PR: promoter region; RET: Rearranged During Transfection; ROS1: ROS Proto-Oncogene 1, Receptor Tyrosine Kinase; SD: structural domain; SLC34A2: Solute Carrier Family 34 Member 2; SDC4: Syndecan 4; TPM3: Tropomyosin.

\section{Results}

\subsection{Panel Design}

The fusion primer pool for SiRe was designed with the AmpliSeq designer software v.7.4.1., which uses hg19 as a reference sequence. Overall, 16 samples in 520 chips on a S5/S5XL Ion Torrent platform (ThermoFisher Scientifics), were run simultaneously. Remarkably, the pool of 91 primer pairs covered all the targeted gene fusions, reaching a $5000 \times$ coverage for each amplicon. Moreover, since the fusion primer pool was fully compatible with the custom NSCLC DNA primer pool, a DNA/RNA pool was generated in a single NGS run. The DNA libraries can be processed in parallel with RNA libraries, under the same thermal and sequencing conditions. The RNA primer pool, as reported in the method section, was developed to be fully integrable with SiRe primers pool [31]. The SiRe results are reported in Supplementary Tables S1 and S2 and Supplementary File S3.

\subsection{Reference Range and Fixation Modalities}

All samples processed for the feasibility test passed the quality filters. The NGS run parameters ensured an optimal analytical performance of the RNA fusion panel, as evidenced by its ability to reliably detect clinically relevant rearrangements in $A L K$, ROS1, $R E T$, and NTRK, as well as MET exon 14 skipping mutations. Taking into account all the mutant cases, we obtained, on average, $99.70 \%$ (ranging from $99.13 \%$ to $99.98 \%$ ) on-target 
reads, $103.55 \mathrm{bp}$ read lengths (ranging from 93.00 to 114.00), 311,798.44 mapped reads (ranging from 60,530.00 to 948,223.00), and 318415.89 coverage uniformity (ranging from 60,562.00 to 96,378.00). The SiRe RNA fusion panel was able to detect $A L K, R O S 1, R E T$, and NTRK rearrangements, as well as MET exon 14 skipping mutations. All the results, including the fusion partner genes, are reported in Supplementary Tables S3-S7.

Adequate quality and quantity RNA and cDNA input were extracted from methanol and ethanol fixed cell lines. Moreover, NGS analysis correctly detected all engineered mutations in both groups (Supplementary Tables S3 and S4) and no false positive results were observed in the wild type groups (Supplementary Tables S5 and S6). The Easy PGX platform further confirmed all the mutations on residual RNA extracted from fusionpositive $A L K, R O S 1$, RET samples, as well as the MET exon 14 skipping kit and the NTRK fusion kit (Supplementary Table S7).

The LoD was determined by using RNA extracted from four cell lines, which were diluted to obtain $50 \%, 25 \%$, and $10 \%$ of translocated EML4-ALK v3 and SLC34A2-ROS1, as reported in the Materials and Methods section. In all instances, the assay was able to detect both ALK and ROS1 fusions.

\subsection{Optimization of Cell Line Quantity and Limit of Detection}

The RNA isolated from all the cell lines (Supplementary Table S2) was pooled to generate five different RNA input quantity points $(20 \mathrm{ng} / \mu \mathrm{L}, 10 \mathrm{ng} / \mu \mathrm{L}, 2 \mathrm{ng} / \mu \mathrm{L}, 0.5 \mathrm{ng} / \mu \mathrm{L}$, $0.1 \mathrm{ng} / \mu \mathrm{L}$ ), and analyzed in the aforementioned manner. Overall, we obtained, on average, $73.97 \%$ (ranging from $64.51 \%$ to $85.60 \%$ ) on-target reads, $93.00 \mathrm{bp}$ (ranging from 48.00 to 117.00 ) read lengths, 21427.80 (ranging from $13,642.00$ to $27,230.00$ ) mapped reads, and 20432.40 (ranging from $28,229.00$ to 13,784.00) coverage uniformity. The SiRe RNA fusion panel was able to detect all fusions and the splicing event harbored by the RNA pool diluted up to $2 \mathrm{ng} / \mu \mathrm{L}$. (Supplementary Table S2).

\subsection{SiRe Fusion Panel in a Routine Setting and Evaluation of Discordant Samples}

The applicability of our SiRe fusion panel to a routine clinical setting was assessed by testing detection performance in 19 cell blocks and 29 histological $(n=15$ resection and $\mathrm{n}=14$ biopsies) fusion positive samples of advanced stage NSCLC patients ( 25 men and 23 women; mean age: 56.0 years) retrieved from the archives. Sample characteristics, mutational status, and technologies are reported in Table 1. Overall, our SiRe fusion panel successfully analyzed 46 (95.8\%) out 48 samples. Among these, 43 (93.5\%) out of 46 samples accurately reproduced the results obtained with conventional techniques. Only three (6.5\%) out of 46 instances yielded discordant results. These discordant results were further confirmed by CE-IVD automated RT-PCR analysis (Easy PGX platform, Diatech Pharmacogenetics) for ALK, ROS1, RET, and NTRK fusions, as well as MET exon 14 skipping mutations. The specific characteristics of the fusions and relative read counts are reported in Table 1.

\section{Discussion}

Advanced lung cancer, like many other malignancies, is driven by various genetic changes, including gene fusions and splicing events. Thus, the identification of therapeutically relevant gene fusions (ALK, ROS1, RET and NTRK) and MET exon 14 skipping mutations is pivotal in choosing correct management of advanced-stage NSCLC [3,6]. Currently, IHC and FISH represent the gold standard methodologies for identifying these alterations, as evidenced in different clinical trials $[5,26]$. However, in a substantial number of cases FISH and IHC yield discordant results [33]. In this setting, RNA-sequencing plays a key role in defining the molecular assessment of tumor samples and in making treatment decisions for patients affected by advanced NSCLC. In addition, the implementation of NGS in routine clinical practice is key to making do with the scant amount of analyzable tissue from these patients, when one considers the increasing number of clinically relevant biomarkers tested in clinical practice. Indeed, NGS enables, not only to test DNA based 
alterations, but also to evaluate relevant gene fusions simultaneously. Today, it is no longer sustainable, especially at the local level, to test clinically relevant biomarkers for NSCLC patients by using IHC-based algorithms or FISH. More recently, the European Society for Medical Oncology (ESMO) recommends the adoption of NGS technology in patient with advanced non-squamous NSCLC to analyze not only DNA-based biomarkers but also RNA-based gene rearrangements and other alterations, such as MET exon 14 skipping mutations. To this end, an integrated RNA- and DNA-based NGS approach should be preferred [34].

In our experience, we demonstrated that our narrow SiRe NGS fusion panel is a reliable tool to detect clinically relevant gene fusions and MET exon 14 skipping alterations in routine samples of lung cancer. Remarkably, our new custom panel accurately reproduced $(93.5 \%)$ the results obtained with conventional techniques. Considering the three discrepant cases between NGS and FISH analysis, it should be considered that in all instances a "borderline" FISH positive results was obtained, featuring a percentage of rearranged nuclei around $15 \%$. In addition, our approach was able to identify all gene fusions and the splicing event harbored in the RNA pool extracted from cell lines, even when the RNA concentration was as low as $2 \mathrm{ng} / \mu \mathrm{L}$. Taking into account this parameter, it is conceivable that the inadequate NGS results may be related to low quality RNA. This underlines its applicability to cytological samples and small histological biopsies, which represent $80 \%$ of all advanced stage NSCLC sample types.

In conclusion, the study fully validates the feasibility of integrating a DNA $\left(\mathrm{SiRe}^{\circledR}\right)$ and RNA (SiRe fusion) primer pool in an Ampliseq based approach to cover all the clinically relevant NSCLC alterations (EGFR, KRAS, BRAF, ALK, ROS1, RET, NTRK, and METex14) simultaneously in a single run, regardless of the small sample size of available tissue. A further study is ongoing to evaluate in addition to the specificity also the sensitivity of our panel on a wide cohort of patients, including not only mutated but also wild type cases.

Supplementary Materials: The following are available online at https://www.mdpi.com/2072 -6694/13/1/139/s1. Supplementary File S1. SiRe fusion panel: design. Supplementary File S2. SiRe fusion panel: software for analysis. Supplementary File S3. SiRe fusion panel: run parameters. Supplementary Table S1. American Type Culture Collection Cell lines used to assess limit of detection and reference range of the SiRe fusion primer pool and relative results obtained with the developed analytical and bioinformatics workflow. Supplementary Table S2. The SiRe fusion results at different dilution points. Supplementary Table S3. Results obtained from FP-MEOH samples. Fused transcripts identified with our NGS approach are highlighted in red. Supplementary Table S4. Results obtained from FP-ETOH samples. The fused transcripts identified with our NGS approach are highlighted in red. Supplementary Table S5. Results obtained from FN-MEOH samples. Supplementary Table S6. Results obtained from FN-ETOH samples. Supplementary Table S7. Fusion positive results obtained from $\mathrm{FP}-\mathrm{MEOH}$ residual RNA samples after storage for one month at $-20^{\circ} \mathrm{C}$. Supplementary Figure S1. Panel A shows the result obtained from RNA extracted from FN-ETOH; Panel B shows the result obtained from cDNA produced from the same sample; Panel C shows the results obtained in RNA extracted from FN-MEOH; Panel D shows the results obtained from cDNA produced from the same sample. Overall, enough quality and the quantity of RNA and relative CDNA was obtained prepare libraries for next-generation sequencing analysis (NGS) by using our SiRe ${ }^{\circledR}$ fusion panel (Department of Public Health, University of Naples Federico II). Supplementary Figure S2. Panel A shows the results obtained from RNA extracted from FP-ETOH; Panel B shows the results obtained from cDNA produced from the same sample; Panel C shows the result obtained from RNA extracted from FP-MEOH; Panel D shows the results obtained from cDNA produced from the same sample. Overall, the quality and the quantity of RNA and relative cDNA were adequate to prepare libraries for next-generation sequencing analysis (NGS) by using our SiRe ${ }^{\circledR}$ fusion panel (Department of Public Health, University of Naples Federico II). 
Author Contributions: Conceptualization: U.M., G.T.; methodology: all authors; software, C.D.L., F.P. (Francesco Pepe), A.I., P.P., L.R., A.L., L.G., G.G., S.C., G.D.D., F.P. (Fabio Pagni), R.S., M.N., R.T., F.C., E.V., C.B., D.L.C., S.N., M.A.M.-V., R.R., G.T., U.M.; validation: C.D.L., F.P. (Francesco Pepe), A.I., P.P., L.R., A.L., L.G., G.G., S.C., G.D.D., F.P. (Fabio Pagni), R.S., M.N., R.T., F.C., E.V., C.B., D.L.C., S.N., M.A.M.-V., R.R., G.T., U.M.; formal analysis: C.D.L., F.P. (Francesco Pepe), A.I., P.P., L.R., A.L., L.G., G.G., S.C., G.D.D., F.P. (Fabio Pagni), R.S., M.N., R.T., F.C., E.V., C.B., D.L.C., S.N., M.A.M.-V., R.R., G.T., U.M.; investigation: C.D.L., F.P. (Francesco Pepe), A.I., P.P., L.R., A.L., L.G., G.G., S.C., G.D.D., F.P. (Fabio Pagni), R.S., M.N., R.T., F.C., E.V., C.B., D.L.C., S.N., M.A.M.-V., R.R., G.T., U.M.; resources: C.D.L., F.P. (Francesco Pepe), A.I., P.P., L.R., A.L., L.G., G.G., S.C., G.D.D., F.P. (Fabio Pagni), R.S., M.N., R.T., F.C., E.V., C.B., D.L.C., S.N., M.A.M.-V., R.R., G.T., U.M.; data curation: C.D.L., F.P. (Francesco Pepe), A.I., P.P., L.R., A.L., L.G., G.G., S.C., G.D.D., F.P. (Fabio Pagni), R.S., M.N., R.T., F.C., E.V., C.B., D.L.C., S.N., M.A.M.-V., R.R., G.T., U.M.; writing—original draft preparation: U.M., C.D.L., F.P. (Francesco Pepe), P.P., G.T.; writing—review and editing: all authors; visualization: all authors; supervision: U.M., G.T.; project administration: U.M., G.T.; funding acquisition: U.M., G.T. All authors have read and agreed to the published version of the manuscript.

Funding: 1. Monitoraggio ambientale, studio ed approfondimento della salute della popolazione residente in aree a rischio-In attuazione della D.G.R. Campanian.180/2019. 2. POR Campania FESR 2014-2020 Progetto “Sviluppo di Approcci Terapeutici Innovativi per patologie Neoplastiche resistenti ai trattamenti-SATIN".

Institutional Review Board Statement: All information regarding human material was managed using anonymous numerical codes, and all samples were handled in compliance with the Declaration of Helsinki (http:/ / www.wma.net/en/30publications/10policies/b3/).

Informed Consent Statement: Written informed consent was obtained from patients and documented in accordance with the general authorization to process personal data for scientific research purposes from 'The Italian Data Protection Authority' (http://www.garanteprivacy.it/web/guest/ home/docweb/- / docwebdisplay / export/2485392).

Data Availability Statement: The data presented in this study are available in the manuscript and in the supplementary files.

Acknowledgments: We thank Paola Merolla for editing the manuscript.

Conflicts of Interest: Umberto Malapelle has received personal fees (as consultant and/or speaker bureau) from Boehringer Ingelheim, Roche, MSD, Amgen, Thermo Fisher Scientific, Diaceutics, GSK, Merck, and AstraZeneca, unrelated to the current work. Luisella Righi has received personal fees (as consultant and/or speaker bureau) from Astra Zeneca, Novartis, Amgen, and Boehringer Ingelheim, unrelated to the current work. Fabio Pagni has received personal fees (as consultant and/or speaker bureau) from, MSD, GSK, Merck, and AstraZeneca, unrelated to the current work. Silvia Novello reports personal fees (as speaker bureau or advisor) from Eli Lilly, MSD, Roche, BMS, Takeda, Pfizer, Astra Zeneca, and Boehringer Ingelheim. Giancarlo Troncone reports personal fees (as speaker bureau or advisor) from Roche, MSD, Pfizer, and Bayer, unrelated to the current work. The other authors declare no potential conflict of interest.

\section{References}

1. Siegel, R.L.; Miller, K.D.; Jemal, A. Cancer statistics, 2020. CA Cancer J. Clin. 2020, 70, 7-30. [CrossRef]

2. Yuan, M.; Huang, L.L.; Chen, J.H.; Wu, J.; Xu, Q. The emerging treatment landscape of targeted therapy in non-small-cell lung cancer. Signal. Transduct. Target. Ther. 2019, 4, 61. [CrossRef] [PubMed]

3. Remon, J.; Ahn, M.J.; Girard, N.; Johnson, M.; Kim, D.W.; Lopes, G.; Pillai, R.N.; Solomon, B.; Villacampa, G.; Zhou, Q. AdvancedStage Non-Small Cell Lung Cancer: Advances in Thoracic Oncology 2018. J. Thorac. Oncol. 2019, 14, 1134-1155. [CrossRef] [PubMed]

4. Bagley, S.J.; Bauml, J.M.; Langer, C.J. PD-1/PD-L1 immune checkpoint blockade in non-small cell lung cancer. Clin. Adv. Hematol. Oncol. 2015, 13, 676-683. [PubMed]

5. Pisapia, P.; Lozano, M.D.; Vigliar, E.; Bellevicine, C.; Pepe, F.; Malapelle, U.; Troncone, G. ALK and ROS1 testing on lung cancer cytologic samples: Perspectives. Cancer Cytopathol. 2017, 125, 817-830. [CrossRef]

6. Politi, K.; Herbst, R.S. Lung cancer in the era of precision medicine. Clin Cancer Res. 2015, 21, 2213-2220. [CrossRef]

7. Pennell, N.A.; Arcila, M.E.; Gandara, D.R.; West, H. Biomarker Testing for Patients with Advanced Non-Small Cell Lung Cancer: Real-World Issues and Tough Choices. Am. Soc. Clin. Oncol. Educ. Book 2019, 39, 531-542. [CrossRef]

8. Okamura, R.; Boichard, A.; Kato, S.; Sicklick, J.K.; Bazhenova, L.; Kurzrock, R. Analysis of NTRK Alterations in Pan-Cancer Adult and Pediatric Malignancies: Implications for NTRK-Targeted Therapeutics. JCO Precis. Oncol. 2018, 2018. [CrossRef] 
9. Soda, M.; Choi, Y.L.; Enomoto, M.; Takada, S.; Yamashita, Y.; Ishikawa, S.; Fujiwara, S.; Watanabe, H.; Kurashina, K.; Hatanaka, H.; et al. Identification of the transforming EML4-ALK fusion gene in non-small-cell lung cancer. Nature 2007, 448, 561-566. [CrossRef]

10. Shaw, A.T.; Kim, D.W.; Nakagawa, K.; Seto, T.; Crinó, L.; Ahn, M.J.; De Pas, T.; Besse, B.; Solomon, B.J.; Blackhall, F.; et al. Crizotinib versus chemotherapy in advanced ALK-positive lung cancer. N. Engl. J. Med. 2013, 368, 2385-2394. [CrossRef]

11. Solomon, B.J.; Mok, T.; Kim, D.W.; Wu, Y.L.; Nakagawa, K.; Mekhail, T.; Felip, E.; Cappuzzo, F.; Paolini, J.; Usari, T.; et al. PROFILE 1014 Investigators. First-line crizotinib versus chemotherapy in ALK-positive lung cancer. N. Engl. J. Med. 2014, 371, $2167-2177$. [CrossRef] [PubMed]

12. Peters, S.; Camidge, D.R.; Shaw, A.T.; Gadgeel, S.; Ahn, J.S.; Kim, D.W.; Ou, S.I.; Pérol, M.; Dziadziuszko, R.; Rosell, R.; et al. ALEX Trial Investigators. Alectinib versus Crizotinib in Untreated ALK-Positive Non-Small-Cell Lung Cancer. N. Engl. J. Med. 2017, 377, 829-838. [CrossRef] [PubMed]

13. Hida, T.; Nokihara, H.; Kondo, M.; Kim, Y.H.; Azuma, K.; Seto, T.; Takiguchi, Y.; Nishio, M.; Yoshioka, H.; Imamura, F.; et al. Alectinib versus crizotinib in patients with ALK-positive non-small-cell lung cancer (J-ALEX): An open-label, randomised phase 3 trial. Lancet 2017, 390, 29-39. [CrossRef]

14. Camidge, D.R.; Kim, H.R.; Ahn, M.J.; Yang, J.C.; Han, J.Y.; Lee, J.S.; Hochmair, M.J.; Li, J.Y.; Chang, G.C.; Lee, K.H.; et al. Brigatinib versus Crizotinib in ALK-Positive Non-Small-Cell Lung Cancer. N. Engl. J. Med. 2018, 379, 2027-2039. [CrossRef]

15. Rikova, K.; Guo, A.; Zeng, Q.; Possemato, A.; Yu, J.; Haack, H.; Nardone, J.; Lee, K.; Reeves, C.; Li, Y.; et al. Global survey of phosphotyrosine signaling identifies oncogenic kinases in lung cancer. Cell 2007, 131, 1190-1203. [CrossRef]

16. Savic, S.; Rothschild, S.; Bubendorf, L. Lonely Driver ROS1. J. Thorac. Oncol. 2017, 12, 776-777. [CrossRef]

17. Shaw, A.T.; Ou, S.H.; Bang, Y.J.; Camidge, D.R.; Solomon, B.J.; Salgia, R.; Riely, G.J.; Varella-Garcia, M.; Shapiro, G.I.; Costa, D.B.; et al. Crizotinib in ROS1-rearranged non-small-cell lung cancer. N. Engl. J. Med. 2014, 371, 1963-1971. [CrossRef]

18. Drilon, A.; Siena, S.; Ou, S.I.; Patel, M.; Ahn, M.J.; Lee, J.; Bauer, T.M.; Farago, A.F.; Wheler, J.J.; Liu, S.V.; et al. Safety and Antitumor Activity of the Multitargeted Pan-TRK, ROS1, and ALK Inhibitor Entrectinib: Combined Results from Two Phase I Trials (ALKA-372-001 and STARTRK-1). Cancer Discov. 2017, 7, 400-409. [CrossRef]

19. Vaishnavi, A.; Capelletti, M.; Le, A.T.; Kako, S.; Butaney, M.; Ercan, D.; Mahale, S.; Davies, K.D.; Aisner, D.L.; Pilling, A.B.; et al. Oncogenic and drug-sensitive NTRK1 rearrangements in lung cancer. Nat. Med. 2013, 19, 1469-1472. [CrossRef]

20. Harada, T.; Yatabe, Y.; Takeshita, M.; Koga, T.; Yano, T.; Wang, Y.; Giaccone, G. Role and relevance of TrkB mutations and expression in non-small cell lung cancer. Clin. Cancer Res. 2011, 17, 2638-2645. [CrossRef]

21. Marchetti, A.; Felicioni, L.; Pelosi, G.; Del Grammastro, M.; Fumagalli, C.; Sciarrotta, M.; Malatesta, S.; Chella, A.; Barassi, F.; Mucilli, F.; et al. Frequent mutations in the neurotrophic tyrosine receptor kinase gene family in large cell neuroendocrine carcinoma of the lung. Hum. Mutat. 2008, 29, 609-616. [CrossRef] [PubMed]

22. Kohno, T.; Ichikawa, H.; Totoki, Y.; Yasuda, K.; Hiramoto, M.; Nammo, T.; Sakamoto, H.; Tsuta, K.; Furuta, K.; Shimada, Y.; et al. KIF5B-RET fusions in lung adenocarcinoma. Nat. Med. 2012, 18, 375-377. [CrossRef] [PubMed]

23. Mendoza, L. Clinical development of RET inhibitors in RET-rearranged non-small cell lung cancer: Update. Oncol. Rev. 2018, 12, 352. [CrossRef] [PubMed]

24. Drilon, A.; Rekhtman, N.; Arcila, M.; Wang, L.; Ni, A.; Albano, M.; Van Voorthuysen, M.; Somwar, R.; Smith, R.S.; Montecalvo, J.; et al. Cabozantinib in patients with advanced RET-rearranged non-small-cell lung cancer: An open-label, single-centre, phase 2, single-arm trial. Lancet Oncol. 2016, 17, 1653-1660. [CrossRef]

25. Li, A.Y.; McCusker, M.G.; Russo, A.; Scilla, K.A.; Gittens, A.; Arensmeyer, K.; Mehra, R.; Adamo, V.; Rolfo, C. RET fusions in solid tumors. Cancer Treat. Rev. 2019, 81, 101911. [CrossRef]

26. Barbareschi, M.; Barberis, M.; Buttitta, F.; Doglioni, C.; Fiorentino, M.; Fontanini, G.; Franco, R.; Marchetti, A.; Rossi, G.; Troncone, G. Predictive markers in lung cancer: A few hints for the practicing pathologist. Pathologica 2018, 110, 29-38.

27. Cohen, D.; Hondelink, L.M.; Solleveld-Westerink, N.; Uljee, S.M.; Ruano, D.; Cleton-Jansen, A.M.; von der Thüsen, J.H.; Ramai, S.R.S.; Postmus, P.E.; Graadt van Roggen, J.F.; et al. Optimizing Mutation and Fusion Detection in NSCLC by Sequential DNA and RNA Sequencing. J. Thorac. Oncol. 2020, 15, 1000-1014. [CrossRef]

28. Haynes, B.C.; Blidner, R.A.; Cardwell, R.D.; Zeigler, R.; Gokul, S.; Thibert, J.R.; Chen, L.; Fujimoto, J.; Papadimitrakopoulou, V.A.; Wistuba, I.I.; et al. An Integrated Next-Generation Sequencing System for Analyzing DNA Mutations, Gene Fusions, and RNA Expression in Lung Cancer. Transl. Oncol. 2019, 12, 836-845. [CrossRef]

29. Davies, K.D.; Lomboy, A.; Lawrence, C.A.; Yourshaw, M.; Bocsi, G.T.; Camidge, D.R.; Aisner, D.L. DNA-Based versus RNA-Based Detection of MET Exon 14 Skipping Events in Lung Cancer. J. Thorac. Oncol. 2019, 14, 737-741. [CrossRef]

30. Williams, H.L.; Walsh, K.; Diamond, A.; Oniscu, A.; Deans, Z.C. Validation of the Oncomine ${ }^{\mathrm{TM}}$ focus panel for next-generation sequencing of clinical tumour samples. Virchows Arch. 2018, 473, 489-503. [CrossRef]

31. Malapelle, U.; Mayo de-Las-Casas, C.; Rocco, D.; Garzon, M.; Pisapia, P.; Jordana-Ariza, N.; Russo, M.; Sgariglia, R.; De Luca, C.; Pepe, F.; et al. Development of a gene panel for next-generation sequencing of clinically relevant mutations in cell-free DNA from cancer patients. Br. J. Cancer 2017, 116, 802-810. [CrossRef] [PubMed]

32. Gu, Z.; Gu, L.; Eils, R.; Schlesner, M.; Brors, B. circlize Implements and enhances circular visualization in R. Bioinformatics 2014, 30, 2811-2812. [CrossRef] [PubMed] 
33. Scattone, A.; Catino, A.; Schirosi, L.; Caldarola, L.; Tommasi, S.; Lacalamita, R.; Montagna, E.S.; Galetta, D.; Serio, G.; Zito, F.A.; et al. Discordance between FISH, IHC, and NGS Analysis of ALK Status in Advanced Non-Small Cell Lung Cancer (NSCLC): A Brief Report of 7 Cases. Transl. Oncol. 2019, 12, 389-395. [CrossRef] [PubMed]

34. Mosele, F.; Remon, J.; Mateo, J.; Westphalen, C.B.; Barlesi, F.; Lolkema, M.P.; Normanno, N.; Scarpa, A.; Robson, M.; MericBernstam, F.; et al. Recommendations for the use of next-generation sequencing (NGS) for patients with metastatic cancers: A report from the ESMO Precision Medicine Working Group. Ann. Oncol. 2020, 31, 1491-1505. [CrossRef] [PubMed] 